\title{
PERCEPÇÃO ACADÊMICA NA ASSISTÊNCIA DE ENFERMAGEM NO CUIDADO INTENSIVO PEDIÁTRICO ${ }^{1}$
}

ACADEMIC PERCEPTION IN NURSING CARE IN PEDIATRIC INTENSIVE CARE

\author{
Patrine Paz Soares², Rafaela Machado Pena de Matos $^{3}$, Carolina Calvo Pereira ${ }^{4}$, \\ Adriana Dall'Asta Pereira ${ }^{5}$ e Carla Lizandra de Lima Ferreira ${ }^{6}$
}

\section{RESUMO}

O estudo trata-se de um relato de experiência acadêmica desenvolvido a partir de uma vivência teórico-prática sobre a assistência de enfermagem em uma unidade de terapia intensiva pediátrica. Tem como objetivo relatar a experiência acadêmica em uma unidade de cuidado intensivo pediátrico acerca da assistência de enfermagem, em um hospital de grande porte na cidade de Santa Maria - RS, no ano de 2018. O enfermeiro que trabalha com intensivismo passa por muitos desafios, principalmente quando se trata de crianças. Vivenciar o cuidado e todo o processo de trabalho é indispensável para a formação do acadêmico de enfermagem, pois esse processo proporciona reflexão e aprendizado, além de fortalecer habilidades técnicas.

Palavras-chave: Cuidados críticos, Cuidados de enfermagem, Unidades de Cuidado Intensivo Pediátrico, Hospitalização.

\section{ABSTRACT}

The study is a report of academic experience, developed from a theoretical-practical experience on nursing care in a pediatric intensive care unit. It aims to report the academic experience in a pediatric intensive care unit about nursing care in a large hospital in the city of Santa Maria-RS, in the year 2018. The intensive care nurse goes through many challenges, especially when it comes to children. Experiencing the care and the whole work process is indispensable for the formation of the nursing academic, as this process provides reflection and learning, besides strengthening technical skills.

Keywords: Critical care, Nursing care, Pediatric Intensive Care Units, Hospitalization.

\footnotetext{
${ }^{1}$ Estudo oriundo do Estágio II do Curso de Enfermagem - Universidade Franciscana

${ }^{2}$ Autor. Enfermeira Residente do Programa de Residência multiprofissional UFSM. E-mail: patrinepsoares@hotmail.com

${ }^{3}$ Autor. Enfermeira. E-mail: rafaelamatos0910@hotmail.com

${ }^{4}$ Autor. Enfermeira. E-mail: calvocarolina2@gmail.com

${ }^{5}$ Autor. Docente do Curso de Enfermagem - Universidade Franciscana. E-mail: adrianadap@terra.com.br

${ }^{6}$ Orientador - Docente do Curso de Enfermagem - Universidade Franciscana. E-mail: carlalizandralferreira@gmail.com
} 


\section{INTRODUÇÃO}

As Unidades de Cuidados Intensivos (UTI) são conhecidas pela sua complexidade. Nesse ambiente, utiliza-se alta tecnologia, procedimentos invasivos e modernos, monitorização constante, ruídos, luzes e circulação de profissionais em maior proporção (FELIPIN et al., 2018).

Para Coelho et al. (2017), as Unidades de Cuidado Intensivo Pediátrico (UTIP) são locais destinados ao tratamento das necessidades de saúde da criança e assistência de elevada complexidade, dispondo de uma equipe capacitada para esse cuidado. A equipe de enfermagem tem competências técnicas e teóricas para desenvolver assistência, organiza o trabalho e planeja as atividades a serem desenvolvidas na assistência à criança, considerando o ambiente intensivo ao qual ela foi inserida (COELHO et al., 2017).

$\mathrm{O}$ adoecimento e a hospitalização geram sentimentos negativos, como medo, ansiedade, insegurança, tanto na criança como nos familiares. Esse processo causa fragilidade e demanda apoio, compreensão e orientação dos profissionais (FERREIRA et al., 2018). O desafio dos profissionais dentro das UTIs é aliar a grande tecnologia existente a um cuidado humanizado e, assim, perceber o paciente de forma holística, utilizando toque terapêutico, atenção e afeto. Nesse contexto, o enfoque não é apenas voltado para a patologia, mas também na assistência com todos os aparatos tecnológicos necessários para o cuidado (ALMEIDA; FÓFANO, 2016).

O acolhimento da criança e da família, assim como a boa relação com a equipe, demonstra a dedicação que o profissional enfermeiro precisa ter para beneficiar a todos que participam desse processo terapêutico. Assim, é preciso que se proporcione assistência singularizada e se busque benefícios e resultados positivos no cuidado, o que torna o enfrentamento de condições estressantes e difíceis mais tranquilo (BRAGA et al., 2015).

O enfermeiro tem a responsabilidade de abordar todos os aspectos tanto da família como da criança, auxiliando no tratamento e recuperação, compartilhando o cuidado com familiares e equipe, de forma humanizada, indo além dos recursos materiais, da complexidade da unidade ou sofisticação dos equipamentos (RAMÍREZ et al., 2018; FRANCHI et al., 2018). A assistência de enfermagem tem o objetivo de auxiliar na recuperação da criança ou minimizar o seu sofrimento. É o profissional que está em contínuo contato com o paciente durante a sua internação, dedicando-se na tentativa de promover o bem-estar físico e emocional (SANTOS; SILVA; PRUDÊNCIO, 2016).

Diante da importância do papel do profissional enfermeiro no cuidado à criança, assistência e processo de trabalho, este estudo tem como objetivo relatar a experiência acadêmica em uma unidade de cuidado intensivo pediátrico acerca da assistência de enfermagem. 


\section{MÉTODO}

Trata-se de um relato de experiência acadêmica, desenvolvido a partir de uma vivência teórico-prática sobre a assistência de enfermagem em uma unidade de terapia intensiva pediátrica, acerca de plano de ensino da disciplina de Estágio II, do curso de Enfermagem da Universidade Franciscana (UFN). Esse estágio tem como objetivo proporcionar ao aluno o conhecimento por meio das experiências práticas nos diferentes campos de atuação do profissional de enfermagem.

A experiência ocorreu no período de outubro a novembro de 2018, em um hospital de grande porte localizado na cidade de Santa Maria - RS, no turno da noite. A UTIP interna crianças com idades que variam entre 29 dias e 16 anos. A unidade conta com oito leitos, sendo dois deles destinados para usuários em isolamento.

A construção do presente estudo ocorreu durante as teorizações de estágio, quando eram discutidas as potencialidades e fragilidades encontradas durante as demandas dos campos de atuação. Com este estudo, pretende-se compartilhar com professores e colegas as experiências vivenciadas e, assim, destacar a importância do aprendizado adquirido com as práticas, auxiliando na assistência prestada aos usuários, no cuidado com a saúde e na formação profissional.

\section{RESULTADOS E DISCUSSÃO}

A hospitalização na infância é um processo complexo que envolve não apenas a criança, mas também a família e a equipe de saúde, principalmente a enfermagem. A internação pode proporcionar o surgimento de sensações de medo, tristeza, ansiedade, entre outros sentimentos que podem afetar negativamente a recuperação da criança, além de dificultar o cuidado de enfermagem (LEITE; VERGILIO; SILVA, 2017).

Durante o período de estágio realizado na UTIP, foi possível observar e prestar assistência a crianças de diferentes idades e patologias. Nesse espaço, evidenciaram-se situações e comportamentos de revolta, tristeza e ansiedade. Alguns se mostravam mais acessíveis aos cuidados de enfermagem, demonstrando tranquilidade e confiança frente às intervenções, porém outros apresentavam choro frequente e agressividade, o que dificultou a comunicação entre equipe e paciente.

Para Silva e Brandão (2017), o brincar no ambiente hospitalar pode auxiliar e amenizar os sentimentos negativos causados pela internação. Ajuda no processo de crescimento e desenvolvimento da criança, além de aproximar a realidade hospitalar do seu cotidiano, o que torna o cuidado de enfermagem mais acessível.

Nesse processo de cuidado e recuperação, a família é fundamental, pois tem a capacidade de beneficiar e contribuir para que a criança enfrente esse processo com mais segurança. $\mathrm{O}$ apoio da equipe de saúde, principalmente do enfermeiro, torna-se importante nesse período, pois assim é 
possível identificar, acolher e esclarecer as dúvidas, reconhecendo a família como parte do cuidado à criança (RAMOS et al., 2016).

O vínculo construído entre a equipe de enfermagem e a família foi observado durante o período de estágio, pois havia demonstração de confiança e carinho na comunicação. Porém, em momentos de angústia e nervosismo, a família, por vezes, apresentava hostilidade, por meio de questionamentos e impaciência, ao tratar com os profissionais.

O enfermeiro que trabalha com intensivismo passa por muitos desafios, principalmente quando trabalha com crianças. As demandas complexas do ambiente necessitam de conhecimento técnico e científico, além do desenvolvimento de atividades não apenas assistenciais, mas também gerenciais no cuidado, o que envolve os aspectos físico e emocional do profissional, pois é preciso lidar com questões éticas e com a humanização da assistência (BRAGA et al., 2015).

A UTIP trata-se de uma unidade de alta complexidade e tem a comunicação como forma primordial para estabelecer o cuidado à criança, mas, durante a pesquisa, muitas não apresentavam condições de comunicar-se verbalmente, ou seja, estavam impossibilitadas de verbalizar suas carências devido a suas condições clínicas ou sua idade. Diante disso, o profissional deve ter olhar crítico e habilidade para interpretar os sinais apresentados, como expressões faciais, agitação ou choro.

A prática em UTIP proporciona ao acadêmico experiências e práticas mais complexas da rotina de cuidado com a criança, o que demanda delicadeza e atenção. Conforme Silva e Brandão (2017), o profissional enfermeiro desempenha muitas atividades, tanto assistenciais como de gerência, em uma unidade intensiva, ligadas direta ou indiretamente ao cuidado, sendo algumas delas privativas da profissão, como cuidados com pacientes graves, passagem de sondas e cateteres, instalações de alguns medicamentos, curativos e a Sistematização da Assistência de Enfermagem (SAE).

Todos os procedimentos e cuidados prestados pela equipe de enfermagem são registrados nos prontuários eletrônicos de cada paciente. O enfermeiro planeja os cuidados a partir das prescrições médicas, das necessidades do paciente pediátrico e, principalmente, por meio da implementação da SAE. O acadêmico, nesse espaço, tem a oportunidade de desenvolver todas as atividades, sejam elas privativas do enfermeiro ou não, buscando por conhecimento e auxiliando a equipe nos cuidados, o que fortalece o vínculo com os pacientes pediátricos e seus familiares.

Os documentos e registros escritos são instrumentos essenciais no processo de cuidado em saúde. Nestes, constam as atividades desenvolvidas que contribuem para o planejamento e a execução dos serviços, visando à identificação das necessidades e especificidades de cada usuário. Por se tratar de um contexto complexo, a elaboração dos registros completos deve ser minuciosa, visto que podem comprometer a comunicação e, consequentemente, trazer riscos para a segurança do paciente (PINHEIRO et al., 2018).

Além dos registros, as demandas assistenciais também precisam de uma atenção redobrada por parte da equipe de enfermagem dentro das unidades de terapia intensiva, visto que a maioria dos 
enfermos está dependente dos serviços prestados. O cuidado com a pele é uma das responsabilidades do enfermeiro, as lesões por pressão não são muito comuns em crianças, mas, quando há falhas no cuidado, elas podem acontecer. Portanto, cabe ao profissional de enfermagem prevenir lesões, avaliá-las, realizar curativos e implementar cuidados, utilizando protocolos, escalas e plano de cuidados voltados para a assistência da criança, juntamente com auxílio da equipe multiprofissional (FERREIRA et al., 2018).

Outra atribuição do profissional enfermeiro na assistência à criança e que se trata de um grande desafio, é obter um acesso venoso para a administração da terapêutica intravenosa, geralmente necessária no período de internação, utilizada para amenizar a dor e efeitos das patologias.

A punção venosa periférica demanda dos profissionais da enfermagem habilidades, como escolha do local mais adequado, inserção do dispositivo, manipulação, avaliação e retirada, visando à eficiência e qualidade, de modo a permanecer durante maior período de tempo. É um dos procedimentos mais realizados em crianças hospitalizadas. É um método doloroso, porém necessário, que visa beneficiar e auxiliar na recuperação da criança (ALMEIDA et al., 2016). Cabe ao enfermeiro realizar vigilância constante para segurança e prevenção de riscos e complicações associadas à punção e terapia endovenosa. Nesse contexto, é importante a execução de alguns cuidados, como a escolha adequada do dispositivo, antissepsia da pele, atentando para os sinais de alteração, bem como a verificação da permeabilidade do cateter antes da administração de fármacos (NAKANDAKARI et al., 2018).

Para o paciente pediátrico, a atenção no que diz respeito à segurança do paciente na assistência hospitalar deve ser aumentada pela equipe de enfermagem, visando à prevenção de quedas, higienização adequada das mãos, identificação correta do paciente, ao preparo e à administração medicamentosa. Com a criança, é preciso ter cuidados especiais, como manter as guardas dos leitos sempre elevadas, a fim de prevenir qualquer tipo de queda. Além disso, é necessário atentar para medidas de controle de infecção tanto entre os profissionais de saúde como entre os acompanhantes. As medidas de proteção e prevenção devem ser constantes na busca de um cuidado resolutivo, associado à responsabilidade da equipe em orientar os acompanhantes sobre os devidos assuntos (BANDEIRA et al., 2017).

Identificar necessidades e amenizar os desconfortos pode favorecer bem-estar e cuidado humanizado à criança. Também é fundamental, nesse processo, quando for possível, falar e escutar o que a criança tem a dizer, partilhar informações e ações, o que favorece o trabalho do profissional e o desenvolvimento do vínculo.

Uma das atividades de grande importância na rotina da equipe de enfermagem é a passagem de plantão. É essencial para a continuidade do cuidado e da segurança do paciente pediátrico. Nesse momento, ocorre o compartilhamento de informações e avaliações, e a comunicação deve ser eficaz entre os membros da equipe (SILVA et al., 2016).

Após a passagem de plantão, o enfermeiro presente no turno era o responsável por dividir os profissionais técnicos de enfermagem e encaminhá-los aos leitos. Os acadêmicos ficavam sob supervisão e acompanhamento desse enfermeiro durante o turno de trabalho, realizando os cuidados 
juntamente com a equipe, desenvolvendo gerência e assistência, cuidados de rotina, como alternância de decúbito, troca de local de sensor de oximetria, diluição e administração de medicações prescritas pelos médicos, banho de leito, manuseio de drenos, verificações de sinais vitais, ventilação mecânica, passagens de sonda nasoenteral e vesical. Também eram responsáveis pela realização da SAE e articulação com os profissionais médicos e fisioterapeutas presentes no setor.

O profissional enfermeiro, além de realizar a assistência direta ao paciente, realiza a gestão do cuidado por meio da SAE. Esse instrumento é privativo do enfermeiro e requer uma reflexão acerca da realidade do local onde ele está inserido. Trata-se de um processo direcionado para o cuidado e assistência à saúde, a partir do qual são feitas intervenções adequadas e individuais conforme a necessidade de cada indivíduo, em busca de resultados e resolutividade (GUEDES; SANTOS; OLIVEIRA, 2017).

A SAE é utilizada para sistematizar e organizar a assistência. Utiliza a coleta de dados para construção de um histórico do paciente, aplicando conhecimento clínico e científico na programação e planejamento das intervenções, após os diagnósticos de enfermagem. Esse instrumento tem contribuição na tomada de decisão, pois evidencia as necessidades e particularidades do indivíduo, além de proporcionar respaldo ao profissional e autonomia no processo de cuidado em saúde (COELHO et al., 2017; SANTOS et al., 2016).

O enfermeiro tem a responsabilidade de organizar e planejar o cuidado a partir das necessidades da criança, buscando melhorar a qualidade da assistência e proporcionar segurança para o paciente. Para o acadêmico de enfermagem, vivenciar o cuidado e todo o processo de trabalho é indispensável para sua formação, pois proporciona reflexão e aprendizado, além de fortalecer habilidades técnicas.

\section{CONSIDERAÇÕES FINAIS}

A hospitalização de uma criança na UTIP é bastante complexa. Esse local apresenta grande aporte tecnológico e procedimentos invasivos, o que distancia a criança de sua rotina, da família, dos amigos e das atividades cotidianas, aspectos que tornam o enfrentamento da criança e dos familiares mais difícil.

$\mathrm{O}$ enfermeiro que atua nesse local tem a responsabilidade de prestar assistência prática e gerenciar o cuidado à criança de forma humanizada, sem focar apenas na patologia, mas no cuidado integral, visando ao bem-estar, à segurança e à proteção, prevenindo eventos adversos que possam comprometer ainda mais a saúde do paciente.

A realização deste estudo proporcionou aprendizagem, habilidade e reflexão teórico-prática sobre os processos de enfermagem, a utilização da SAE, os procedimentos realizados na UTIP, assim como medidas de segurança do paciente, demandas assistenciais e gerenciais e desafios enfrentados pelo profissional enfermeiro ao desempenhar seu trabalho no cuidado intensivo.

$\mathrm{O}$ acadêmico de enfermagem na UTIP aprende sobre as normas e rotinas da unidade, realiza técnicas e procedimentos, desenvolve a gerência da unidade e do processo de trabalho. Além disso, 
atua nas atividades de baixa e alta complexidade, compartilha conhecimento com a equipe e vincula-se com a criança e a família. Pela sua complexidade, é um ambiente de muito aprendizado, que possibilita a reflexão crítica e humanizada durante a graduação.

\section{REFERÊNCIAS}

ALMEIDA, T. J. C.; MIRANDA, J. O. F.; SANTOS, L. M.; SANTANA, R. C. B.; CAMARGO, C, L.; SOBRINHO, C. L. N. Acessos venosos periféricos em crianças hospitalizadas: um estudo fotográfico. Revista de enfermagem UFPE [online], Recife, v.10, Supl. 2, p. 701-7, fev., 2016.

ALMEIDA, Q.; FÓFANO, G. A. Tecnologias leves aplicadas ao cuidado de enfermagem na unidade de terapia intensiva: uma revisão de literatura. HU Revista, Juiz de Fora, v. 42, n. 3, p. 191-196, set-out., 2016.

BANDEIRA, L. E.; WEGNER, W.; GERHARDT, L. M.; PASIN, S. S.; PEDRO, E. N. R.; KANTORSKI, K. J. C. Condutas de educação ao familiar para promoção da segurança da criança hospitalizada: registros da equipe multiprofissional. REME - Revista Mineira de Enfermagem, v. 21. 2017.

BRAGA, L. C.; SOUSA, F. G. M.; SANTOS, M. H.; SANTOS, D. M. A. Demandas de atenção do enfermeiro em unidade de terapia intensiva pediátrica: uma investigação qualitativa. Arquivos de Ciências da Saúde, v. 22, n. 4, p. 52-57, out-dez., 2015.

COELHO, A. V; MOLINA, R. M; LABEGALINI, M. P. C.; ICHISATO, S. M. T.; PUPULIM, J. S. L. Validação de um histórico de enfermagem para unidade de terapia intensiva pediátrica. Revista Gaúcha Enfermagem, v. 38, n. 3, 2017.

FELIPIN, L. C. S.; MERINO, M. F. G. L.; BAENA, J. A.; OLIVEIRA, R. B. S. R.; BORGHESAN, N. B. A.; HIGARASHI, I. H. Cuidado centrado na família em unidade de terapia intensiva neonatal e pediátrica: visão do enfermeiro. Ciência, Cuidado e Saúde, v. 17, n. 2, abr-jun., 2018.

FERREIRA, M. J. M.; DODT, R. C. M.; LIMA, A. M.; M. D. R. F.; PINHEIRO, S. M. P. R. Percepção dos acompanhantes sobre dispositivos invasivos em uma unidade de terapia intensiva pediátrica. Enfermagem em Foco, v. 9, n. 2, p. 18-22, 2018.

FERREIRA, M. K. M.; GURGEL, S. S.; LIMA, F. E. T.; CARDOSO, M. V. L. M. L.; SILVA, V. M. Instrumentos para cuidado de lesão por pressão na pediatria e hebiatria: revisão integrativa da literatura. Revista Latino-Americana de Enfermagem, v. 26, p. 3034, 2018. 
FRANCHI, R.; IDIARTE, L.; DARRIGOL, J.; PEREIRA, L.; SUÁREZ, R.; MASTROPIERRO, L.; FERNÁNDEZ, A. Unidad de cuidados intensivos pediátricos de puertas abiertas: experiencia y opinión de los padres. Arch Pediatr Urug [online], v. 89, n. 3, p. 165-170, 2018.

GUEDES, D. M. B.; SANTOS, L. C. E.; OLIVEIRA, E. A. Intervenções de enfermagem em uma unidade de terapia intensiva pediátrica. Revista de enfermagem UFPE [online], Recife, v. 11, n. 1, p. 102-111, jan., 2017.

LEITE, T. M. C.; VERGíliO M. S. T. G.; SILVA E. M. Processo de trabalho do enfermeiro pediatra: uma realidade a ser transformada. Revista Rene, v. 18, n. 1, p. 26-34, jan-fev., 2017.

NAKANDAKARI, R. A.; BALIEIRO, M. M.; ANACLETO, A. S.; KUSAHARA, D. M.; AVELAR, A. F. Nursing practices related to peripheral intravenous catheterization in newborns and children. Rev Soc Bras Enf Ped, v. 18, n. 1, p. 29-36, 2018.

PINHEIRO, J. M. S.; SOUSA, F. G. M.; SILVA, A. C. O.; SIlVA, N. M. C. E.; SANTOS, D. M. A. Manejo do histórico de enfermagem no contexto da terapia intensiva pediátrica. Revista de Enfermagem da UFSM, v. 8, n. 2, p. 350-365, abr-jun., 2018.

RAMÍREZ, M.; NAVARRO, S.; CLAVERÍA, C.; MOLINA, Y.; COX, A. Estresores parentales en Unidad de Cuidados Intensivos Pediátricos. Revista Chilena de Pediatría, v. 89, n. 2, p. 182-189, 2018.

RAMOS D. Z.; LIMA, C. A.; LEAL, A. L. R.; PRADO, P. F; OLIVEIRA, V. V.; SOUZA, A. A. M.; FIGUEIREDO, M. L.; LEITE, M. T. S. A participação da família no cuidado às crianças internadas em unidade de terapia intensiva. Revista Brasileira em Promoção da Saúde, Fortaleza, v. 29, n. 2 , p. 189-196, abr-jun., 2016.

SANTOS, D. M. A.; SOUSA, F. G. M.; PAIVA, M. V. S.; SANTOS, A. T.; PINHEIRO J. M. S. A enfermagem baseada em evidências apoiando a construção do histórico de enfermagem: uma pesquisa bibliográfica. Ciência, Cuidado e Saúde, v. 15, n. 3, p. 561-569, jul-set., 2016.

SANTOS, F. C.; SILVA, I. C.; PRUDÊNCIO, F. A. Papel da Enfermagem e da Família na Assistência e Recuperação da Criança Hospitalizada. Revista Saúde em Foco, Teresina, v. 4, n. 1, art. 5, p. 58-66, jan-jul., 2017. 
SILVA, D. F; BRANDÃO, E. C. As práticas lúdicas no cotidiano do cuidar em enfermagem pediátrica. REVACI, Brasília, v. 2, n. 2, jan-jul., 2017.

SILVA, M. F.; ANDERS, J. C.; ROCHA, P. K.; SOUZA, A. I. J.; BURCIAGA, V. B. Comunicação na passagem de plantão de enfermagem: segurança do paciente pediátrico. Texto \& Contexto Enfermagem, v. 25, n. 3, p. 1-9, 2016. 
